\title{
REFERENCES
}

1. Lee EW, Saab S, Kaldas F, et al. Coil-assisted retrograde transvenous obliteration (CARTO): an alternative treatment option for refractory hepatic encephalopathy. Am J Gastroenterol. 2018;113:1187-96.

2. Mukund A, Deogaonkar G, Rajesh S, et al. Safety and efficacy of sodium tetradecyl sulfate and lipiodol foam in balloon-occluded retrograde transvenous obliteration (BRTO) for large porto-systemic shunts. Cardiovasc Interv Radiol. 2017;40:1010-6.

3. Kim YH, Kim YH, Kim CS, et al. Comparison of balloon-occluded retrograde transvenous obliteration (BRTO) using ethanolamine oleate (EO), BRTO using sodium tetradecyl sulfate (STS) foam and vascular plug-assisted retrograde transvenous obliteration (PARTO). Cardiovasc Interv Radiol. 2016;39:840-6.

4. Luo X, Ma H, Yu J, et al. Efficacy and safety of balloon-occluded retrograde transvenous obliteration of gastric varices with lauromacrogol foam sclerotherapy: initial experience. Abdom Radiol. 2018;43:1820-4.

5. Lee EW, Saab S, Gomes AS, et al. Coil-assisted retrograde transvenous obliteration (CARTO) for the treatment of portal hypertensive variceal bleeding: preliminary results. Clin Transl Gastroenterol. 2014;5:e61.

6. Kim DJ, Darcy MD, Mani NB, et al. Modified balloon-occluded retrograde transvenous obliteration (BRTO) techniques for the treatment of gastric varices: vascular plug-assisted retrograde transvenous obliteration (PARTO)/coil-assisted retrograde transvenous obliteration (CARTO)/ balloon-occluded antegrade transvenous obliteration (BATO). Cardiovasc Interv Radiol. 2018;41:835-47.

Department of Gastroenterology and Hepatology, West China Hospital, Sichuan University, No. 37 Guoxue Alley, Wuhou District, Chengdu, Sichuan Province, 610041, China. Correspondence: L.Y. (email: yangli_hx@scu.edu.cn)

\section{Response to Yang et al.}

\author{
Edward Wolfgang Lee, $\mathrm{MD}, \mathrm{PhD}^{1,2,3}$ and \\ Stephen T. Kee, MD ${ }^{1,2}$
}

Am J Gastroenterol https://doi.org/10.1038/s41395018-0385-0

To the Editor: We appreciate the authors of this commentary showing an interest in our study. Our study was not intended to demonstrate the superiority of coilassisted retrograde Transvenous obliteration (CARTO). We believe that all three (BRTO, CARTO, and PARTO) treatment options should be available at those insti- tutions that treat the sequelae of portal hypertension.

Given the complexity of overt hepatic encephalopathy (OHE), we believe it will be challenging to achieve $100 \%$ clinical success to reverse their symptoms. Therefore, there is no perfect treatment option available for these patients yet. We believe a more significant number of patients is needed to understand and validate the true effects of CARTO or BRTO in OHE patients. Also, the success rate of CARTO (91\%)[1] is in the similar range or better than other published studies (60-100\%)[2-4]. It would be extremely difficult and challenging to state that one technique is better than others with a small number of patients and in a non-prospective manner.

Concerning gelfoam slurry vs. sclerosing agent, several studies have demonstrated the effectiveness of gelfoam slurry in the treatment of gastric varices and other portosystemic shunts with minimal complications and high clinical and technical efficacy [1, 2, 5-7]. Gelfoam may not cause permanent endothelial destruction; however, it does cause complete thrombosis with long-term follow-up shown in both CARTO and PARTO [1, 2, 5-7]. Lauromacrogol (polidocanol) has been shown to be effective [8-11]; however, there have been several reports of complications associated with its use [8-10].

If one analyzes the cost of supplies utilized in CARTO/PARTO vs. BRTO, CARTO/PARTO may be more expensive than BRTO as coils and plugs are more expensive than balloons in most institutions. However, if the overall cost of the procedure(s) is considered including (1) the cost of additional [2, 3] procedures in the angio suite required in BRTO to check the status of shunt/varices sclerosis (2), the cost of an ICU, or higher level of care room, required due to the pressure of an indwelling BRTO balloon (3), the cost of sclerosing agent (e.g., Sotradecol $(\mathrm{STS})=\$ 450$ US vs. gelfoam $=\$ 16$ US) and (4) the potential cost due to more severe complications caused by BRTO, we firmly believe the CARTO/PARTO can be cost-effective. However, no comparison study or prospective data are available to validate this.
Regarding the procedure time, again, a single procedure time of CARTO may be longer than BRTO or PARTO as it requires additional time to deploy multiple coils as stated in our study. However, if the overall procedure time is analyzed including the time of additional procedures as mentioned above, we believe that CARTO will be compared favorably to BRTO.

In summary, we firmly believe that the modified BRTO (CARTO/PARTO) has a vital role in managing patients with complications of portal hypertension, including gastric variceal bleeding and overt hepatic encephalopathy. Multiple studies have demonstrated its clinical efficacy and safety. CARTO has some distinct advantages in treating patients with large portosystemic shunts and very tortuous vascular anatomy. Many are reluctant to keep patients with balloons inflated in situ for prolonged periods of time. Also, potential rare complications from displaced sclerosants have presented BRTO from becoming a widely accepted procedure in the U.S. and Western countries. The relative safety of CARTO along with the operator comfort and familiarity associated with the use of coils and gelfoam provide an opportunity for many patients to benefit from this procedure in the future.

\section{CONFLICT OF INTEREST}

Guarantor of the article: Edward Wolfgang Lee, MD, PhD.

Specific author contributions: Edward Wolfgang Lee-Planning, drafting, revision and approving of the final manuscript, Stephen T Kee-Planning, Revision and approving of the final manuscript.

\section{Financial support: None.}

Potential competing interest: None.

\section{REFERENCES}

1. Lee EW, Saab S, Kaldas F, et al. Coil-assisted retrograde transvenous obliteration (CARTO): an alternative treatment option for refractory hepatic encephalopathy. Am J Gastroenterol. 2018;113:1187-96.

2. Gwon DI, Kim YH, Ko GY, et al. Vascular Plug assisted retrograde transvenous obliteration for the treatment of gastric varices and hepatic encephalopathy: a prospective multicenter study. J Vasc Interv Radiol. 2015;26:1589-95.

3. Laleman W, Simon-Talero M, Maleux G, et al. Embolization of large spontaneous portosystemic shunts for refractory hepatic encephalopathy: a multicenter survey on safety and efficacy. Hepatology. 2013;57:2448-57. 
4. Mukund A, Rajesh S, Arora A, et al. Efficacy of balloon-occluded retrograde transvenous obliteration of large spontaneous lienorenal shunt in patients with severe recurrent hepatic encephalopathy with foam sclerotherapy: initial experience. J Vasc Interv Radiol. 2012;23:1200-6.

5. Chang MY, Kim MD, Kim T, et al. Plug-assisted retrograde transvenous obliteration for the treatment of gastric variceal hemorrhage. Korean J Radiol. 2016;17:230-8.

6. Kim T, Yang H, Lee CK, et al. Vascular plug assisted retrograde transvenous obliteration (PARTO) for gastric varix bleeding patients in the emergent clinical setting. Yonsei Med J. 2016;57:973-9.

7. Lee EW, Saab S, Gomes AS, et al. Coil-assisted retrograde transvenous obliteration (CARTO) for the treatment of portal hypertensive variceal bleeding: preliminary results. Clin Transl Gastroenterol. 2014;5:e61.

8. Choi SY, Won JY, Kim KA, et al. Foam sclerotherapy using polidocanol for balloonoccluded retrograde transvenous obliteration (BRTO). Eur Radiol. 2011;21:122-9.

9. Clements W, Cavanagh K, Ali F, et al. Variant treatment for gastric varices with polidocanol foam using balloon-occluded retrograde transvenous obliteration: a pilot study. J Med Imaging Radiat Oncol. 2012;56:599-605.

10. Itou C, Koizumi J, Hashimoto T, et al. Balloon-occluded retrograde transvenous obliteration for the treatment of gastric varices: polidocanol foam versus liquid ethanolamine oleate. Am J Roentgenol. 2015;205:659-66.

11. Luo X, Ma H, Yu J, et al. Efficacy and safety of balloon-occluded retrograde transvenous obliteration of gastric varices with lauromacrogol foam sclerotherapy: initial experience. Abdom Radiol. 2018;43:1820-24.

'Division of Interventional Radiology, Department of Radiology, UCLA Medical Center, David Geffen School of Medicine at UCLA, Los Angeles, CA, USA. ' $U C L A$ Pfleger Liver Institute, University of California at Los Angeles, Los Angeles, CA, USA. ${ }^{3}$ Dumont-UCLA Transplant Center, Division of Liver and Pancreas Transplantation, Department of Surgery, David Geffen School of Medicine at University of California, Los Angeles, Los Angeles, CA, USA. Correspondence: E.W.L. (email:

310.267.8771EdwardLee@mednet.ucla.edu)

\section{Anti-TNF}

\section{Exposure in} Utero, What About My Twins?

\author{
Sunanda Kane, MD, MSPH and \\ Brenda Becker, CCRP
}

Am J Gastroenterol https://doi.org/10.1038/s41395018-0165-x
To the Editor: We read with great interest the study by Chaparro et al. in regard to outcomes of infants exposed to anti-TNF in utero [1]. In their study, they found no increased risk for serious infections in those infants exposed to anti-TNF agents in utero compared with non-exposed. However, they did find a higher proportion of complications in terms of cesarean sections, need for ICU admission and low birth weight.

This is important work that adds to our body of knowledge with a large cohort followed for more than just a few weeks. In their analysis the authors found that it was pre-term birth, and not the use of anti-TNF therapy that was associated with an increased risk of adverse birth outcomes. These results should be comforting to our patients, who are concerned about the risks of continued biologic exposure during pregnancy.

But as with other large studies on pregnancy to date this study was limited to only singleton pregnancies. As the number of women undergoing assisted reproductive technology (ART) increases, we clinicians get the obvious question: what will happen to my twins? There are known increased risks for both maternal and neonatal outcomes in multiple gestation pregnancies, but hard to counsel anxious patients without data. We do have some recent population-based data as to the outcomes of twins in the healthy population [2]; future multicenter experience with multiple gestation pregnancies will be just as important to study as the singleton pregnancies.

\section{CONFLICT OF INTEREST}

Guarantor of the article: Sunanda Kane, MD, MSPH.

Specific author contributions: SK: concept of article, writing and review; BB: writing of article and review.

Financial support: None.

Potential competing interests: SK is a consultant to AbbVie, Merck, Samsung Bioepis and UCB. BB declares that she has no conflict of interest.

\section{REFERENCES}

1. Chapparo M, Verreth A, Lobaton T, et al. Long term safety of in utero exposure to anti-TNF alpha drugs for the treatment of inflammatory bowel disease: results from the Multicenter European TEDDY Study. Am J Gastroenterol. 2018;113:396-4-3.
2. Luke B, Gopal D, Cabral H, Stern J, Diop H. Adverse pregnancy, birth and infant outcomes in twins: effects of maternal fertility status and infant gender combinations; the Massachusetts Outcomes Study of Assisted Reproductive Technology. Am J Obstet Gynecol. 2017;217: 330. ei-e15

Mayo Clinic, Rochester, MN, USA.

Correspondence: S.K. (email: kane.sunanda@ mayo.edu)

\section{Pregnancy and Inflammatory Bowel Disease: A Long Way
to Go}

María Chaparro, MD, PhD and Javier P. Gisbert, MD, PhD

Am J Gastroenterol https://doi.org/10.1038/s41395018-0379-y

To the editor: We thank Drs. Kane and Becker for their interest on the TEDDY study [1, 2]. Our study aimed to know the long-term safety of anti-TNF agents in children born to mothers with inflammatory bowel disease (IBD). The safety of these drugs during pregnancy has been demonstrated; however, little is known about their impact on children outcomes. For that reason, we hope that TEDDY study could bring some data to mothers and clinicians to make evidence-based decisions.

However, we think that the TEDDY study is only the first step in a long way to go. IBD affects patients in their reproductive years. To maintain disease in remission is crucial for pregnancy outcome. In the previous years, new biologics have been approved for the treatment of IBD: vedolizumab for Crohn's disease and ulcerative colitis, and ustekinumab for Crohn's disease. Recently, a JAK inhibitor-tofacitinib-has been approved for ulcerative colitis. In the near future, new drugs are expected to increase the therapeutic armamentarium of IBD. All these 